\title{
Anomalous Dispersion and Attractive Pulse Interaction in the 1,4-Cyclohexanedione Belousov-Zhabotinsky Reaction ${ }^{\dagger}$
}

\author{
Chad T. Hamik, Niklas Manz,,$*$ and Oliver Steinbock* \\ Florida State University, Department of Chemistry, Tallahassee, Florida 32306-4390
}

Received: January 23, 2001; In Final Form: April 17, 2001

\begin{abstract}
We report the formation of stable bound wave packets in a modified Belousov-Zhabotinsky reaction. These densely stacked structures arise from an attractive interaction between oxidation pulses that is not known from the classical Belousov-Zhabotinsky system. The characteristic stacking period increases with the initial concentration of bromate but decreases with cyclohexanedione. Wave stacking can also induce cascades of bunching events in which internally dense but mutually well segregated wave clusters are formed. For different initial concentrations, we observed the apparent merging of waves in front-to-back collisions. All three modes of wave dynamics are analyzed in terms of their dispersion behavior. The dispersion relations proved to be anomalous in each case and revealed the existence of an attractor which induces the formation of stable wave packets. The underlying mechanism has a pure reaction-diffusion character since wave propagation is not affected by fluid convection. At high initial concentrations of ferroin, we detected complex relaxation kinetics which indicate the presence of at least two independent species that control the recovery and hence the dispersion behavior of the medium.
\end{abstract}

\section{Introduction}

Propagating waves of excitation are a common phenomenon in a variety of systems far from thermodynamical equilibrium. ${ }^{1-3}$ In contrast to their linear counterparts in electrodynamics and acoustics, excitation waves have constant amplitude and show no interference behavior. These and other unusual characteristics arise from the spatial coupling of local, nonlinear processes. In many chemical and biological systems, the underlying nonlinearity is caused by autocatalysis that can spread through an extended system via the diffusion of the corresponding autocatalytic species. ${ }^{3-5}$ The list of such reaction-diffusion systems ${ }^{6}$ is long and includes the oxidation of $\mathrm{CO}$ on platinum surfaces, ${ }^{7}$ the corroding of metals, ${ }^{8}$ glycolysis, ${ }^{9}$ and even complex biological media such as cultured networks of glial cells ${ }^{10}$ or colonies of Dictyostelium discoideum, ${ }^{11}$ in which traveling waves of cyclic AMP organize the aggregation of thousands of cells.

Another widely studied experimental system is the BelousovZhabotinsky (BZ) reaction. ${ }^{2,4-6}$ This reaction involves the oxidation of an organic substrate by bromate in an acidified aqueous medium. It is catalyzed by appropriate metal ions or metal-ion complexes. The intermediate $\mathrm{HBrO}_{2}$ is the autocatalytic species which determines the propagation velocity of the front according to its rate of production and its diffusion constant. The recovery process in the wake of the front is controlled by a reactive decrease in bromide concentration, which leads the system from the oxidized (i.e., refractory) back into the reduced (i.e., excitable) state.

In a periodic wave train the propagation velocity depends also on the distance between successive wave fronts. ${ }^{12-15}$ For excitation waves, this dispersion relation is often expressed as

$\dagger$ This paper is dedicated to Naresh Dalal on the occasion of his 60th birthday.

* Corresponding author.

$\doteqdot$ Current address: Universität Magdeburg, Institut für Experimentelle Physik, Universitätsplatz 2, D-39106 Magdeburg, Germany. the wavelength or period dependence of the velocity. It reflects the fact that the rate of $\mathrm{HBrO}_{2}$ production is strongly influenced by the concentration of the control variable $\left(\mathrm{Br}^{-}\right.$in the $\mathrm{BZ}$ reaction) at the position of the front. This concentration is determined by the distance between the front and its predecessor and by the relaxation kinetics in the wave back. In the case of a simple monotonic decay, the propagation velocity of a pulse train with very long wavelength equals the velocity of a solitary pulse. However, the velocity decreases for decreasing wavelengths down to a critical value below which no propagation is possible. This most common form of the dispersion relation has been observed experimentally for various systems ${ }^{16-20}$ and is therefore referred to as normal dispersion.

However, even simple two-species reaction-diffusion models can give rise to nonmonotonic kinetics in the wake of an excitation pulse. ${ }^{21-26}$ This surprising behavior can be understood by analyzing the stability of the steady state. For this purpose, the eigenvalues of the Jacobian matrix are derived from the rate laws. ${ }^{5}$ In a typical excitable medium, the eigenvalues are negative real numbers, which indicates that the fixed point is a stable node. In the vicinity of a Hopf bifurcation point, however, the real parts of the eigenvalues vanish, and the stable node can transform to a stable spiral. Hence, the main excitation pulse is followed by damped oscillations in the concentration of both chemical species. A discussion of the conditions for which oscillatory relaxation develops in simple reaction-diffusion models can be found in ref 25 .

A profound consequence of nonmonotonic relaxation is the appearance of an overshoot or ripples in the corresponding dispersion relation. ${ }^{25,26}$ Elphick et al. ${ }^{23}$ distinguished three main types of dispersion relations that are illustrated in Figure 1. In the case of normal dispersion (Figure 1a), a solitary wave is the only stable finite solution. Finite wave trains will continuously increase their relative distances although this trend will become less and less pronounced in the course of propagation. Figure 1b,c shows a sketch of nonmonotonic (or anomalous) 


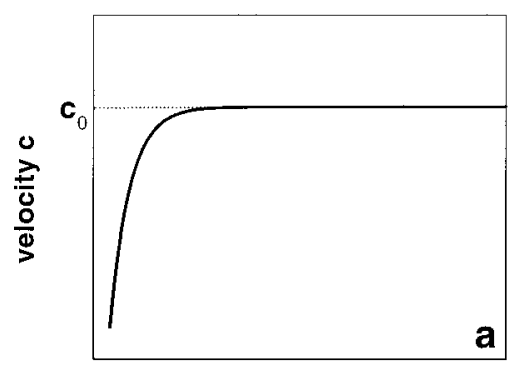

wavelength $\lambda$
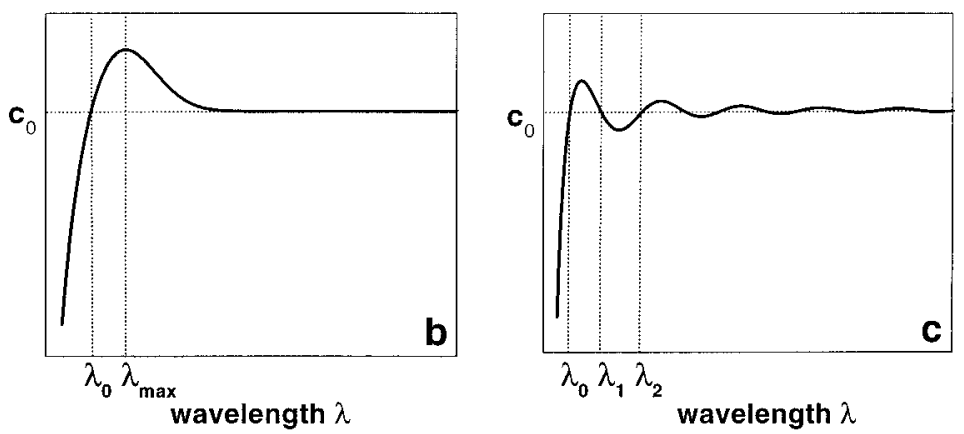

Figure 1. Schematic drawing illustrating three qualitatively different types of dispersion curves: (a) normal, (b) nonmonotonic nonoscillatory, and (c) oscillatory. The velocity $c_{0}$ equals the speed of the solitary wave. Similar curve shapes are found for the propagation velocity as a function of interpulse period.

dispersion relations in which the nonoscillatory nonmonotonic case (Figure 1b) is distinguished from oscillatory one (Figure 1c). In both cases, stable finite wave trains do exist for a discrete spectrum of interpulse distances. The corresponding stability criteria are $\mathrm{c}(\lambda)=c_{0}$ and $\mathrm{d} c / \mathrm{d} \lambda>0$, with $\lambda$ and $c_{0}$ denoting the distance between successive pulses and the velocity of the solitary front, respectively.

Deviations from normal dispersion behavior have been observed only for a small number of experimental systems. Siegert and Weijer reported an isolated event during the aggregation of the slime mold Dictyostelium discoideum that might indicate the possibility of anomalous dispersion in this system. ${ }^{20,27}$ Christoph et al. presented results on propagating waves during the reduction of $\mathrm{NO}$ with $\mathrm{CO}$ on a $\mathrm{Pt}(100)$ surface that are in agreement with front dynamics expected for a reaction-diffusion medium with anomalous dispersion. ${ }^{28}$ More recently, our laboratory reported evidence for the existence of anomalous dispersion in a BZ system where the classic organic substrate malonic acid is replaced by 1,4-cyclohexanedione (CHD). ${ }^{29}$

In this paper, we present a detailed experimental analysis of the wave dynamics in thin capillary tubes filled with CHDBZ solutions. Here, waves spontaneously initiate at the ends of the capillary tubes which are left open to the ambient atmosphere. The overall goal is to establish this reaction as an experimental model for the investigation of anomalous dispersion in excitable media. We provide concrete evidence for attractive interaction between successive pulses that either induces the formation of stable bound wave packets or the apparent merging of pulses in back-to-front collisions. Furthermore, we demonstrate the existence of an instability in which an initially periodic wave train decays into an aperiodic one ("pulse bunching"). This phenomenon was first predicted by Rinzel and Maginu ${ }^{12}$ in 1984 but had not been observed in experiments yet.

\section{Experimental Section}

All reagents are of the highest grade commercially available and used without further purification. Stock solutions of sodium bromate (Fluka) and 1,4-cyclohexanedione (Aldrich) are prepared by dissolved weight of chemical in Nanopure water. Ferroin solution (Fluka, puriss. p.a.; $25 \mathrm{mM}$ ) and sulfuric acid (Fisher; $10.00 \pm 0.05 \mathrm{~N}$ ) are used without further preparation. Working solutions are prepared by additive volumes of stock solutions to give appropriate final concentrations. The stock solutions are always aliquoted in the following order: (1) water, (2) sulfuric acid, (3) 1,4-CHD, (4) sodium bromate, and (5) ferroin. The temperature of the stock solutions and the reaction system is kept constant at $25 \pm 1{ }^{\circ} \mathrm{C}$.
All experiments are carried out in thin capillary tubes. The inner diameter and the length of the capillaries are 1.1 and 75 $\mathrm{mm}$, respectively, with the exception of those in Figure 5, where the inner diameter was varied between $180 \mu \mathrm{m}$ and $1.1 \mathrm{~mm}$. The capillary tube is the reaction vessel of choice in order to create a quasi-one-dimensional system and to minimize perturbations that arise from ambient molecular oxygen. In contrast to the classical $\mathrm{BZ}$ reaction that employs malonic acid as its substrate, the $\mathrm{CHD}-\mathrm{BZ}$ reaction does not generate significant concentrations of gaseous products such as $\mathrm{CO}_{2}$. Consequently, the spatial homogeneity of our experimental system is not compromised by the formation of gas bubbles. ${ }^{30,31}$

The samples are illuminated with diffusive white light to avoid undesired reflection from the curved surface of the capillary tube. The optical contrast in our reaction medium arises from the different absorption spectra of the redox couple ferroin/ ferriin $\left(\left[\mathrm{Fe}(\text { phen })_{3}\right]^{2+} /\left[\mathrm{Fe}(\text { phen })_{3}\right]^{3+}\right) .^{32}$ Experiments are monitored using an image acquisition system that consists of an IBMcompatible PC, a frame grabber board (Data Translation; 640 $\times 480$ pixels resolution with 8 bit/pixel) and a monochrome solid-state camera (COHU 2122). The frame grabber board is controlled by commercial software (HLImage++97). For most experiments, the digital images are acquired with a sampling rate of 0.5 frames/s and a spatial resolution of at least $0.1 \mathrm{~mm} /$ pixel. The resulting data are analyzed after completion of the experiment using homemade software that was developed in an IDL programming environment (Research Systems, Inc.; version 5.1.1).

The data collection is started toward the end of the induction period. At this time, the reaction system switches from the spatially homogeneous oxidized state into the reduced (excitable) state. Notice that the induction time in the CHD-BZ system is rather long and varies with the initial concentrations between 20 and $50 \mathrm{~min}$. The times found here show good agreement with data reported by Kurin-Csörgei et al. ${ }^{33}$ In addition, we observed that the induction time is slightly shortened at the open ends of the capillary tube where the reaction solution is in contact with the air. This small effect stems from a loss of bromine and/or an inflow of oxygen, which is well-known for the classical BZ reaction. ${ }^{34}$ However, due to the length of the capillary tubes $(7.5 \mathrm{~cm})$ and the relatively short duration of our experiments $(<1 \mathrm{~h})$, we can rule out the possibility of a diffusion-controlled contamination of the main reaction medium. This conclusion was also verified by experiments in which the capillaries were sealed off from the ambient atmosphere.

\section{Results}

Dispersion-Induced Stacking, Merging, and Bunching of Waves. Figure 2 shows typical time-space plots that illustrate 

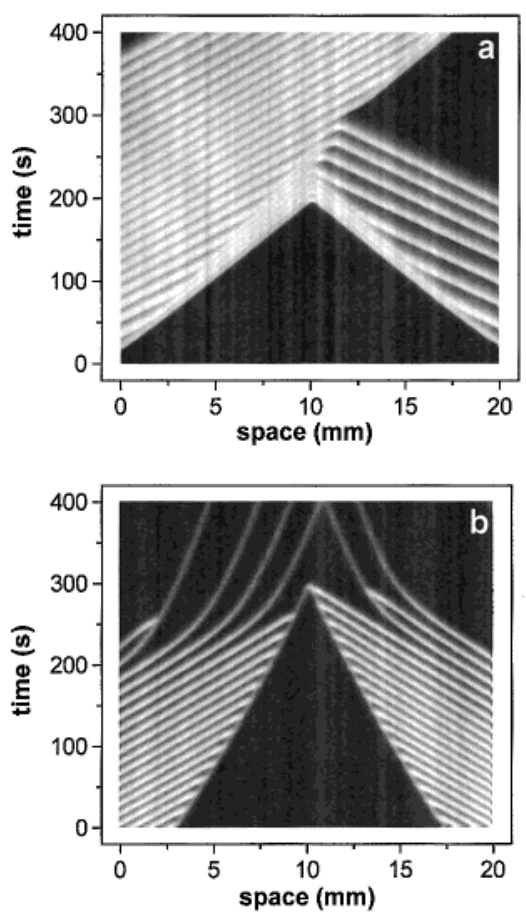

Figure 2. Time-space plots of wave trains in one-dimensional CHD$\mathrm{BZ}$ systems, which exhibit attractive pulse interaction. The figure shows representative examples for the stacking (a) and merging (b) of waves. The two experiments are carried out at different initial concentrations of bromate and cyclohexanedione: $\left[\mathrm{H}_{2} \mathrm{SO}_{4}\right]=2.0 \mathrm{M}$, [ferroin $]=0.5$ $\mathrm{mM},[1,4-\mathrm{CHD}]=0.11$ (a) and $0.25 \mathrm{M}(\mathrm{b})$, and $\left[\mathrm{NaBrO}_{3}\right]=0.07$ (a) and $0.12 \mathrm{M}(\mathrm{b})$.

the wave dynamics in quasi-one-dimensional $\mathrm{CHD}-\mathrm{BZ}$ systems. The two experiments are carried out at different initial concentrations of 1,4-cyclohexanedione and bromate. The timespace plots are produced by piling up spatial absorption profiles at a constant sampling rate. The vertical and horizontal axes represent time and space, respectively. It should be noted that the time scales of the plots solely mark the time range for the given picture but do not reflect the total reaction time. Dark and bright regions indicate reduced and oxidized states, respectively. The plots show white bands that correspond to different propagating oxidation pulses. Their propagation velocities equal the inverse slope of the bands. In both experiments, the capillary tubes were left open to the ambient atmosphere. This procedure facilitates the spontaneous nucleation of oxidation waves from the far ends of the reaction medium.

Figure $2 \mathrm{a}$ is an example of the dynamics that we will refer to as wave stacking. Stacked waves appear as bright, triangular regions in which thin dark (i.e., reduced) stripes separate the individual pulses. The largest stacking pattern in Figure 2a can be found around the coordinates $x=7.5 \mathrm{~mm}$ and $t=150 \mathrm{~s}$. The figure shows that oxidation waves are spontaneously initiated at the open ends of the capillary tube. The leading wave propagates into a fully excitable medium that had not been oxidized for several minutes. The second wave travels at a higher velocity compared to that of the first wave, which leads to a sequence of events that is somewhat analogous to traffic jam created by a "Sunday driver". As the second wave travels at its higher velocity, it draws in on the first wave and continues to approach the first wave until it reaches a characteristic wavelength (here, $0.47 \mathrm{~mm}$ ). Stacking occurs as the second wave's velocity quickly decreases to, and finally matches, that of the first wave. This process is repeated as more waves approach the closely stacked wave packet and, in turn, are themselves incorporated into the wave packet.

It can be seen that waves enter the field of view from both the right and left ends of the capillary tube. Once these waves meet in the central region of the system, they mutually annihilate. Notice that the first pair of colliding waves propagates with the same, relatively slow speed of $3.3 \mathrm{~mm} / \mathrm{min}$. In this experiment, the waves entering the field of observation from the left-hand side of Figure 2a happen to show a higher frequency than those entering from the right. Furthermore, the process of wave initiation ceases spontaneously after eight leftward waves and 24 rightward waves were triggered. Consequently, the last waves entering the system from the left no longer have partners to annihilate with and continue to traverse into a recovered media, where the leading one quickly assumes the slow velocity of the stacked wave packets. This process induces a new sequence of stacking events in the upper right-hand side of the plot. The fact that the velocity of the resulting wave packet equals the velocity of the earlier one shows that the reaction medium is spatially homogeneous and neither affected by temporal transients nor by spatial gradients along the capillary tube.

Figure $2 \mathrm{~b}$ illustrates a typical example of a phenomenon that will be referred to as wave merging. Again, waves nucleate spontaneously at the ends of the capillary tube and begin to move inward. The waves which follow the first front, propagate with a higher velocity, and, thus, approach the back of the leading pulse. In this case, stacking does not occur, but rather, a merging into the leading wave is observed. Careful inspection of numerous time-space plots revealed no evidence for reduced (dark) gaps that would separate the merging bands during frontto-back collisions. However, this finding does not rule out the possibility of a thin repulsion zone in the wake of the pulses because the corresponding control species is not necessarily the optically detected redox couple ferroin/ferriin. Moreover, the existence of an inhibitor barrier in the wake of an excitation pulse appears to be a necessity since it establishes the directionality of wave propagation.

The waves in the upper portion of time-space plot (Figure $2 b$ ) form arch-like bands which indicate a slow but continuous decrease in propagation velocity. This observation demonstrates that waves can escape from the attractive interaction that emanates from their fast predecessors. In the experiment presented, this behavior results from a steady increase in the period of the incoming wave trains. The asymptotic speed of the "escapees" is approximately equal to the speed of the first wave that traveled through the capillary tube.

The measurement of the involved dispersion relations is crucial for a more thorough understanding of the observed phenomena. For excitation waves, this dispersion relation is often expressed as the period (or wavelength) dependence of the wave velocity. ${ }^{25}$ One should distinguish between two different types of dispersion relations. ${ }^{12}$ The first one expresses the velocity of an infinite wave train as a function of its constant period. These data can be obtained from solitary waves in ringshaped systems. The second type of dispersion relation is extracted from the dynamic behavior of fronts that undergo repulsive or attractive interaction with their predecessor. This type of dispersion is analyzed in the following experiments. For simplicity, we will use the term "period" as a synonym for the time elapsed between the passage of subsequent waves through a given position in the medium.

Panels b and d of Figure 3 show two typical examples of dispersion relations that govern the behavior of stacking and 

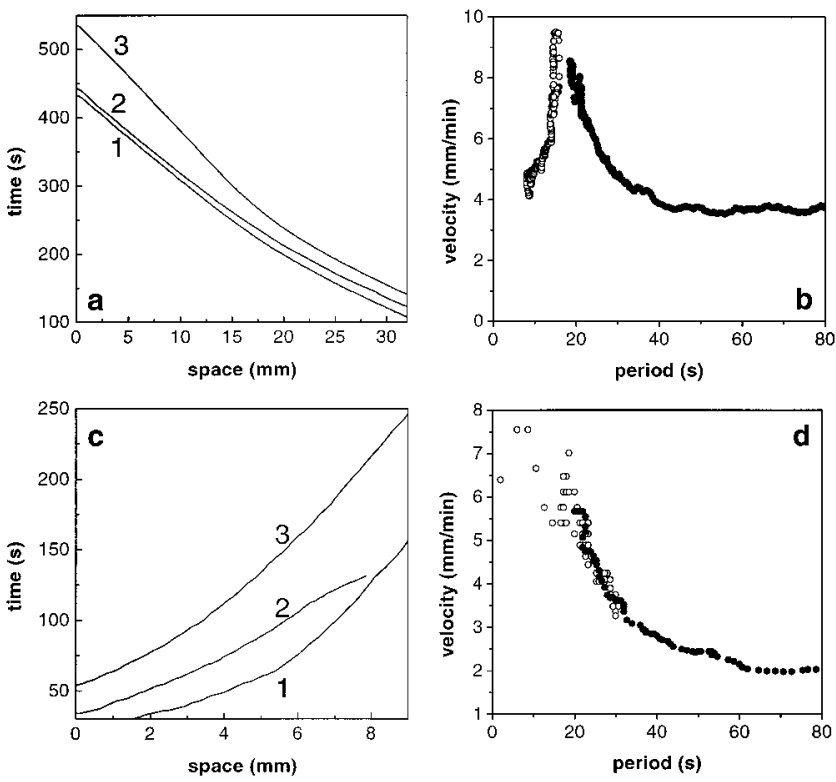

Figure 3. Typical dispersion relations of waves in the CHD-BZ reaction. The dispersion curves $(b, d)$ are obtained from the data shown in the time-space plots $(\mathrm{a}, \mathrm{c})$, respectively. Open and solid circles denote data obtained from the wave pairs $(1,2)$ and $(2,3)$, respectively. Initial concentrations: $\left[\mathrm{H}_{2} \mathrm{SO}_{4}\right]=2.0 \mathrm{M}$, [ferroin] $=0.5 \mathrm{mM},[1,4$ $\mathrm{CHD}]=0.15(\mathrm{a}, \mathrm{b})$ and $0.25 \mathrm{M}(\mathrm{c}, \mathrm{d})$, and $\left[\mathrm{NaBrO}_{3}\right]=0.1(\mathrm{a}, \mathrm{b})$ and $0.12 \mathrm{M}(\mathrm{c}, \mathrm{d})$.

merging waves, respectively. They are obtained by numerical differentiation of the time-dependent positions of three subsequent fronts (Figure 3a,c). The use of three fronts allows the analysis of a wider range of periods since the dynamics of a given single pair is often restricted to either attractive or repulsive interaction. In both dispersion curves, open and solid circles represent data from the wave pairs 1,2 and 2,3, respectively. Accordingly, the velocities are extracted only from the fronts 2 and 3. These velocities partially overlap, which indicates a satisfying degree of consistency in the data.

Figure 3a,b is obtained from a system in which wave stacking occurs. The underlying attractive interaction induces the formation of a bound state between pulses 1 and 2 . The corresponding segment of the dispersion curve (open circles) has a positive slope (i.e., $\mathrm{d} c / \mathrm{d} T>0$ ). In the course of propagation, the pulse pair moves along the dispersion curve toward smaller periods. This relaxation leads to a steady period of $T_{0}=7 \mathrm{~s}$ and a steady velocity of $c_{0}=4.0 \mathrm{~mm} / \mathrm{min}$, which denote a stable state because for these values the slope of the dispersion curve is positive and the velocity equals the one of a solitary wave (i.e., $\mathrm{T} \rightarrow \infty)$.

The initial period between the second and third front in Figure $3 \mathrm{a}$ is longer than the one between fronts 1 and 2 . This small difference is sufficient to induce a decrease in the velocity of wave 3 and therefore a continuous increase in the distance between the waves. The resulting data is represented in Figure $3 \mathrm{~b}$ as solid circles. It should be noted that propagation velocities below $4 \mathrm{~mm} / \mathrm{min}$ have not been observed in this medium but are likely to exist. They are expected for very dense, unstable wave trains that rapidly increase their period to reach the stable value of $T_{0}=7 \mathrm{~s}$.

The data presented in Figure 3c,d are obtained from merging waves. Here, the dispersion relation has a negative derivative, $\mathrm{d} c / \mathrm{d} T$, down to very small periods where the slope possibly becomes positive. The most striking difference from the dispersion relation in Figure $3 b$, however, is that even at very small periods, the velocity is greater than the characteristic value

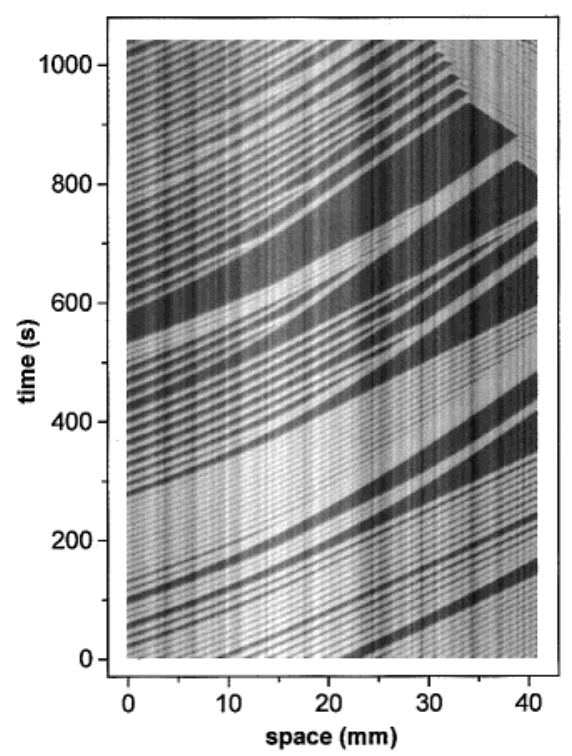

Figure 4. Time-space plot of bunching waves. The system undergoes a cascade of stacking events that yield several internally stable wave clusters spaced at marginally unstable distances. Initial concentrations: $\left[\mathrm{H}_{2} \mathrm{SO}_{4}\right]=2.0 \mathrm{M},[$ ferroin $]=0.1 \mathrm{mM},[1,4-\mathrm{CHD}]=0.11 \mathrm{M}$, and $\left[\mathrm{NaBrO}_{3}\right]=0.10 \mathrm{M}$.

of a solitary wave (here $c_{0}=2 \mathrm{~mm} / \mathrm{min}$ ). The dispersion curve in Figure $3 \mathrm{~d}$ starts at about 3 times the value of $c_{0}$. This difference is the primary source of merging since all fronts that are subject to attractive interaction fail to propagate in the immediate vicinity of their predecessor.

In summary, the above findings show that wave stacking and wave merging result from anomalous dispersion relations. The main feature of systems with wave stacking is the existence of an attractor $\left(c_{0}, T_{0}\right)$ on the dispersion curve, which is characterized by the stability criteria $\mathrm{d} c / \mathrm{d} T>0$ and $c\left(T_{0}\right)=c_{0} \cdot{ }^{12,26} \mathrm{In}$ systems with wave merging, however, such a point does not exist, and the dispersion curve starts prematurely at a high value of velocity.

Exactly one stable type of finite wave trains exists if the dispersion relation has the main characteristics as in Figure 3b. In these wave trains, all interpulse periods must equal $T_{0}$, but the number of pulses is arbitrary. Consequently, wave packets are unstable if they have at least one interpulse period that differs from the value $T_{0}$. Nevertheless, wave trains with very long periods are exceedingly less unstable since the slope of the dispersion curve converges to zero for large periods. The latter statement applies to the stacking as well as the merging of waves and has profound consequences on the behavior of any compound wave train in which the average initial period is larger than the period, $T_{\max }$, at which the dispersion relation reaches its maximal velocity. Theoretical studies by Rinzel, Maginu, and others predict that such wave trains decay into a complex, nonperiodic wave pattern. ${ }^{12,21-23}$ During this decay, impulses group together to form successively larger, and therefore less unstable, bunches. It should be noted that due to the lack of reaction systems with anomalous dispersion, the phenomenon of wave bunching had never been observed in experiments. In the $\mathrm{CHD}-\mathrm{BZ}$ reaction, however, this phenomenon is frequently encountered. A typical example for the bunching of waves in this system is shown in Figure 4. Here, nearly all of the waves are initiated at the left end of the capillary tube (not shown), and the frequency of initiation drifts toward lower values. Because of Rinzel's wave train instability, the initially continuously spaced pattern undergoes a cascade of bunching events. 
a
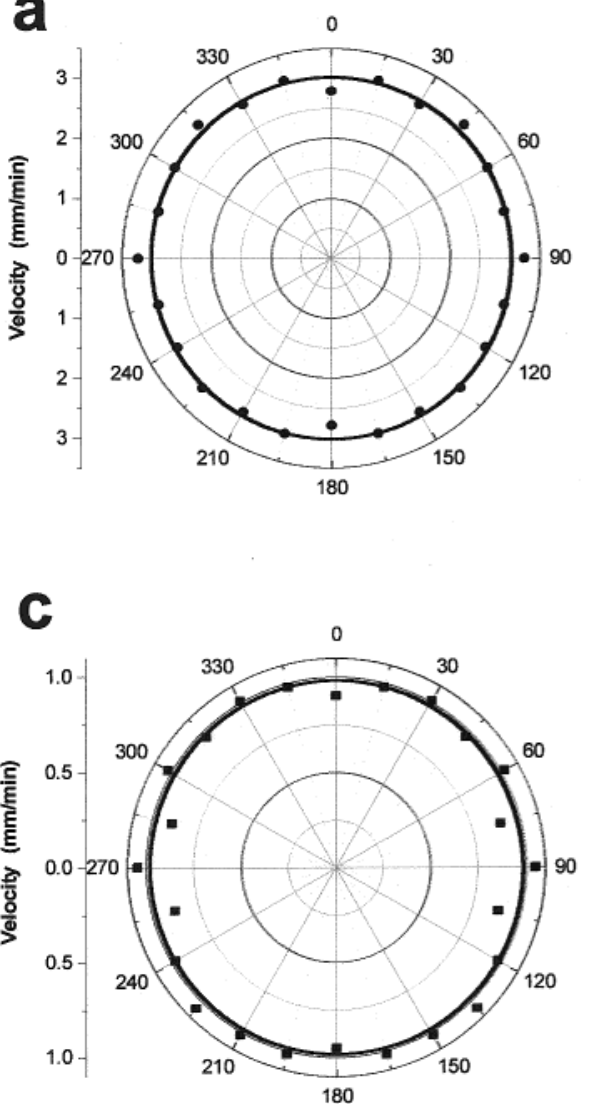

b

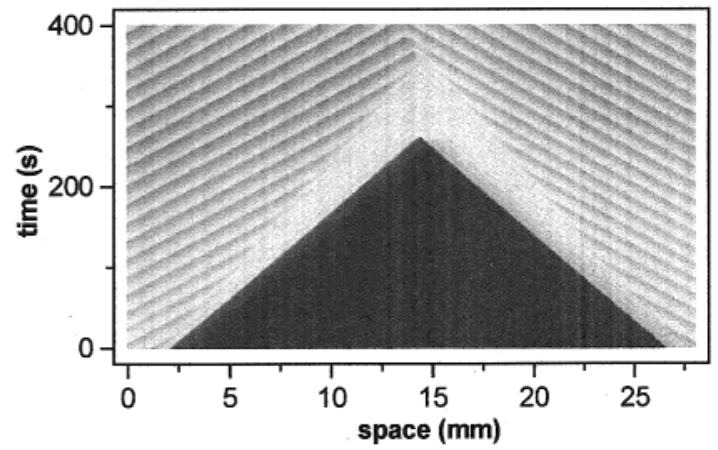

d

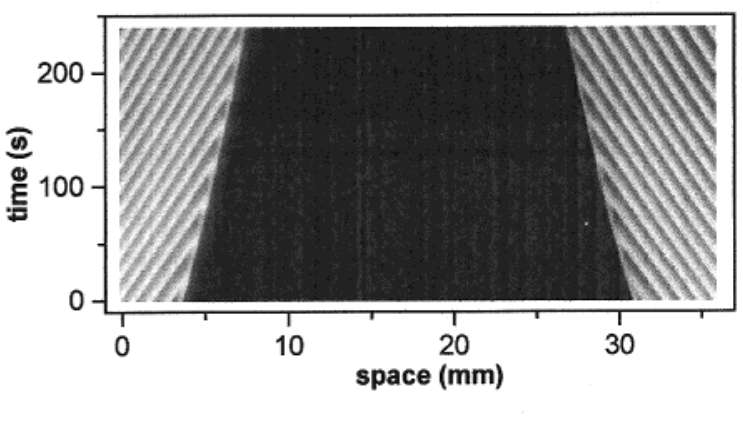

Figure 5. (a, c) Polar coordinate plots of the initial wave velocities vs tube orientations. The $0^{\circ}$ and $180^{\circ}$ positions denote the direction of vertically ascending and vertically descending waves, respectively. The time-space plots $(b, d)$ provide examples for the persistence of attractive pulse interaction in vertical capillary tubes. The initial concentrations in panels a and $\mathrm{b}$ yield wave stacking and the ones in panels $\mathrm{c}$ and $\mathrm{d}$ wave merging: $\left[\mathrm{H}_{2} \mathrm{SO}_{4}\right]=2.0 \mathrm{M}$, [ferroin $]=0.5 \mathrm{mM},\left[\mathrm{NaBrO}_{3}\right]=0.06 \mathrm{M}$, and $[1,4-\mathrm{CHD}]=0.09(\mathrm{a}, \mathrm{b})$ and $0.13 \mathrm{M}(\mathrm{c}, \mathrm{d})$. Inner diameter of the capillary tubes: $1.1 \mathrm{~mm}$.

In each of these events, more and more pulses are incorporated into closely stacked, and therefore stable, wave packets, and the distance between those packets is increased to less unstable values. We encourage the reader to compare Figure 4 with Rinzel and Maginu's theoretical calculation (Figure 6a in ref 12) since the degree of similarity between the numerical and experimental results is striking.

Probing for Conceivable Three-Dimensional Effects and Convection. It is known that pattern formation in the CHD$\mathrm{BZ}$ reaction can be affected by hydrodynamic disturbances that couple to the dominant reaction-diffusion dynamics of the system. ${ }^{35}$ Therefore, we carried out numerous tests that evaluate the role of reaction-induced fluid convection in our experiments. Note that convection in self-organizing chemical systems is not necessarily destructive. Diewald and Brand, ${ }^{36}$ for example, predicted a hydrodynamic stabilization of Turing patterns, and Komlósi et al. ${ }^{35}$ reported a pronounced gravitational anisotropy of wave propagation in the CHD-BZ reaction. The latter authors showed that the speed of reduction waves is significantly increased if the reduction pulse descends through large, vertical tubes (inner diameter $1-8 \mathrm{~mm}$ ). The hypothesis that convection of the $\mathrm{CHD}-\mathrm{BZ}$ solution induces the attraction between oxidation pulses seems therefore noteworthy but will be refuted by the following results.

The possibility of surface-tension-induced fluid flow (e.g., Marangoni convection ${ }^{37}$ ) can be excluded since the reaction system is fully miscible and constrained to solid capillary tubes. If convection cells exist at all, they would arise from differences in the local density of the reaction solution and the resulting buoyancy forces. To investigate the role of buoyancy-driven convection, we varied the tube orientation systematically. The orientation dependence of the overall wave speed and the persistence of stacking and merging behavior are summarized in Figure 5. The polar plots show the wave speed as a function of the angle between the capillary tube and the gravitational field vector. The experiments were performed using one set of concentrations that gives rise to wave stacking (Figure 5a,b), and another set of concentrations that gives rise to the wave merging (Figure 5c,d). The angle was varied in increments of $15^{\circ}$ with $0^{\circ}$ denoting waves that travel upward through a vertical capillary tube. Accordingly, an angle of $180^{\circ}$ denotes downward propagation, and all values are symmetric with respect to the vertical axis of the plot. Each data point (solid symbols) is the average of several, constant-angle experiments, and the thick, solid circle represents the velocity average over all directions. Neither of the polar plots shows any indication for an orientation dependence of the propagation velocity. This result convincingly attests to the absence of buoyancy-driven convection. Panels $b$ and $\mathrm{d}$ of Figure 5 depict time-space plots from merging and stacking experiments, respectively, in which the capillary tubes are in the vertical orientation. The time-space plots reemphasize that the propagation velocity is unaffected by gravity and demonstrate that stacking and merging dynamics persist if the capillary tube is in its most vulnerable, vertical position.

The size and shape of convection cells in a liquid system is intimately related to the size of its container. Komlósi et al. observed that the velocity of reduction waves in the CHD-BZ system can increase by a factor of $4-10$ as the inner diameter 

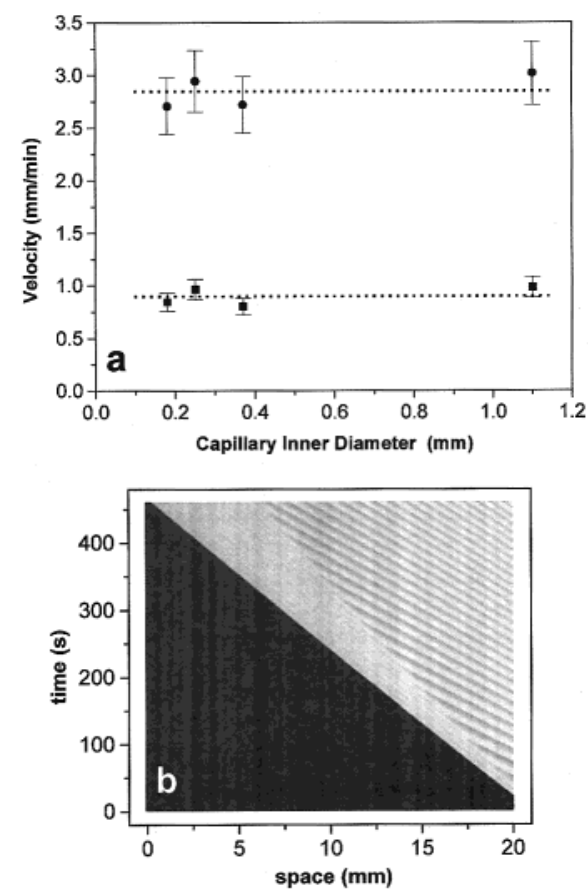

Figure 6. (a) Initial wave velocities as a function of tube diameter for stacking and merging waves. Initial concentrations: $\left[\mathrm{H}_{2} \mathrm{SO}_{4}\right]=2.0$ $\mathrm{M}$, [ferroin] $=0.5 \mathrm{mM},\left[\mathrm{NaBrO}_{3}\right]=0.06 \mathrm{M}$, and $[1,4-\mathrm{CHD}]=0.09$ (circles/stacking) and $0.13 \mathrm{M}$ (squares/merging). (b) Stacking waves in a thin capillary tube (inner diameter $0.37 \mathrm{~mm}$ ). The initial concentrations equal the ones of stacking waves in panel a.

of a cylindrical reaction vessel is changed from $1 \mathrm{~mm}$ to $8 \mathrm{~mm} .{ }^{35}$ Figure $6 \mathrm{a}$ shows the velocity of oxidation waves for different diameters, with the largest one being identical to our standard value of $1.1 \mathrm{~mm}$. The three smaller values of inner diameter are 180, 250, and $370 \mu \mathrm{m}$. The error bars in Figure 6a reflect a relative error of $10 \%$. The results show that there is no dependence on the diameter in this range and that the choice of $1.1 \mathrm{~mm}$ tubes is appropriate. Wave stacking and wave merging is observed for all of the above diameters. Figure $6 \mathrm{~b}$ is a timespace plot of stacking waves recorded in a $370 \mu \mathrm{m}$ tube and is shown as proof of this point. On the basis of these observations, we can rule out the possibility of a hydrodynamic origin of the observed wave dynamics.

Concentration Dependencies. The initial concentrations of bromate and CHD were varied systematically to obtain insights into the chemical mechanism that gives rise to the stacking and merging phenomena and to provide adequate data for subsequent experimental and theoretical studies. Figure 7 a shows a phase diagram of the dispersion-induced wave dynamics, with the ordinate and abscissa representing the initial concentrations of sodium bromate and 1,4-cyclohexanedione, respectively. The initial concentrations of sulfuric acid (2.0 M) and ferroin (0.5 $\mathrm{mM}$ ) are kept constant. Reaction media that allow the propagation of oxidation waves are represented by solid symbols. In the phase diagram, these media form an island surrounded by unexcitable systems that are either oxidized (open diamonds) or reduced (open circles). The relative location of these two particular phase domains reflects the strong oxidizing and reducing effects of bromate and $\mathrm{CHD}$, respectively, and is reminiscent of the classical $\mathrm{BZ}$ reaction that employs malonic acid as its organic substrate. ${ }^{38}$ Figure $7 \mathrm{~b}$ shows a phase diagram similar to the one in Figure $7 \mathrm{a}$ but denotes the according propagation velocities of the leading waves in units of $\mathrm{mm} /$ min. Each numeric value is centered around its corresponding pair of concentrations. The data reveal a pronounced increase
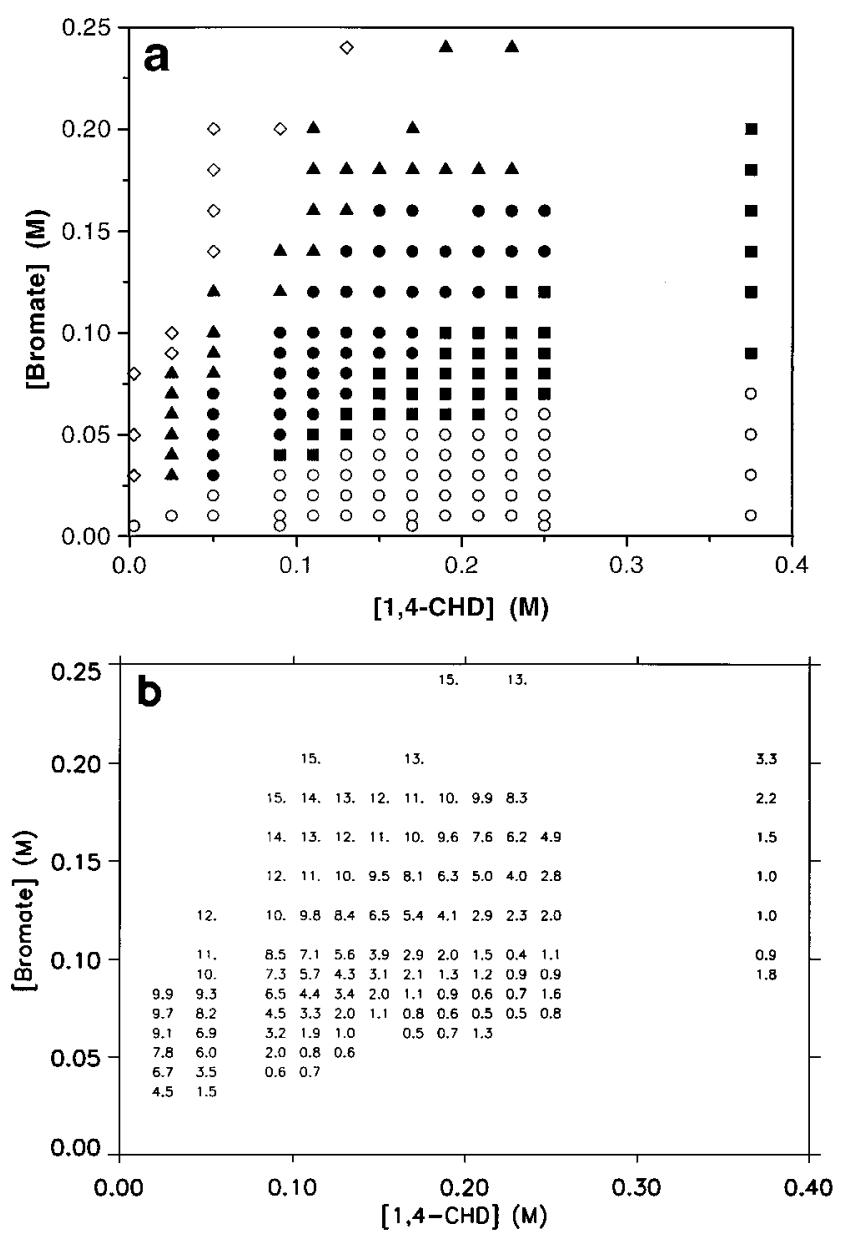

Figure 7. Phase diagrams of the wave dynamics in the bromate-CHD concentration plane. (a) Open diamonds and open circles denote oxidized and reduced states, respectively. Solid squares, circles, and triangles denote cases of merging, stacking, and normal dispersion, respectively. (b) Propagation speed of solitary waves in $\mathrm{mm} / \mathrm{min}$. Initial concentrations: $\left[\mathrm{H}_{2} \mathrm{SO}_{4}\right]=2.0 \mathrm{M}$ and $[$ ferroin $]=0.5 \mathrm{mM}$.

in velocity with increasing concentrations of bromate and decreasing concentrations of CHD. Under the given experimental conditions, the smallest and largest velocities are 0.5 $\mathrm{mm} / \mathrm{min}$ and $15.0 \mathrm{~mm} / \mathrm{min}$, respectively. These values fall into the typical range for BZ-type waves, thus, indicating that the diffusion and the autocatalytic production of $\mathrm{HBrO}_{2}$ cause the propagation of oxidation waves in the $\mathrm{CHD}-\mathrm{BZ}$ reaction.

Figure 7a further distinguishes between systems with qualitatively different types of dispersion behavior. The solid squares indicate concentrations for which wave merging is observed. This type of front interaction is found for relatively small concentrations of bromate but becomes increasingly proliferate at high concentrations of CHD. The experimental conditions that induce wave stacking have consistently higher concentrations of bromate and are represented by solid circles. At even higher bromate concentrations, we found no concrete evidence for anomalous dispersion. Most likely, these parameters (solid triangles in Figure 7a) give rise to systems with normal dispersion. However, we cannot completely rule out the possibility that these systems show wave stacking with very large values of the stable period $T_{0}$, which would imply very broad normal branches of the overall anomalous dispersion curves.

A comparison of panels $\mathrm{a}$ and $\mathrm{b}$ of Figure 7 reveals an interesting correlation between the propagation velocity and the corresponding dispersion behavior. The phase boundaries 

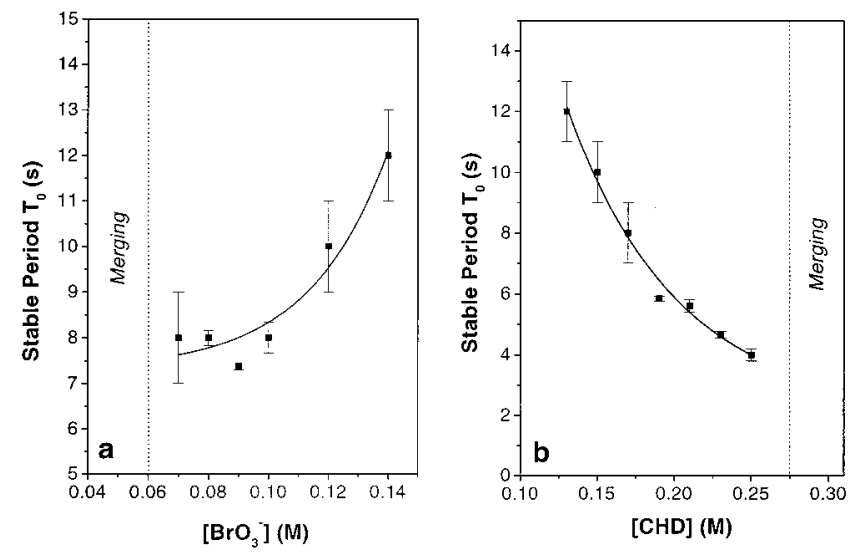

Figure 8. Periods of stacked wave trains as a function of the initial concentration of bromate (a) and 1,4-cyclohexanedione (b). These periods characterize the unique stable wave trains in the corresponding systems. The stacking/merging bifurcation is indicated by the vertical, dotted lines. Initial concentrations: $\left[\mathrm{H}_{2} \mathrm{SO}_{4}\right]=2.0 \mathrm{M}$, [ ferroin $]=0.5$ $\mathrm{mM},[\mathrm{CHD}]=0.13 \mathrm{M}(\mathrm{a})$, and $\left[\mathrm{NaBrO}_{3}\right]=0.14 \mathrm{M}(\mathrm{b})$.

between merging, stacking, and normal dynamics follow roughly the isovelocity lines of solitary wave propagation. This correlation appears to be characteristic for the CHD-BZ system. In the context of Figure 7, for example, wave merging is observed for systems in which a solitary oxidation pulse propagates not faster than $3-5 \mathrm{~mm} / \mathrm{min}$. Wave stacking, on the other hand, is found for velocities below $8-11 \mathrm{~mm} / \mathrm{min}$.

It should also be noted that we occasionally observed transitions from stacking to merging dynamics. These interesting transitions were typically detected for reaction systems close to the stacking/merging boundary. For a given sample, the transition occurs only once and at a reproducible instant. These findings suggest that the transitions arise from the transient nature of the closed reaction system.

The main feature of wave stacking is the existence of a stable period $T_{0}$. Figure 8 shows the concentration dependence of this period with respect to bromate and 1,4-cyclohexanedione. In the underlying experiments, the concentrations of ferroin and sulfuric acid are kept constant at $0.5 \mathrm{mM}$ and $2.0 \mathrm{M}$, respectively. Figure $8 \mathrm{a}$ reveals that the period $T_{0}$ decreases with decreasing concentration of bromate. This smallest value of 8 $\pm 1 \mathrm{~s}$ is reached at a critical concentration of $0.06 \mathrm{M}$. At lower concentrations of bromate, wave stacking changes abruptly to wave merging.

At a concentration of $\left[\mathrm{BrO}_{3}^{-}\right]=0.14 \mathrm{M}$, the period has increased to a value of $12 \pm 1 \mathrm{~s}$. Up to now, we could not obtain reliable data on the wave interaction for higher concentrations of bromate. It is therefore unclear whether the period $T_{0}$ continuous to increase for $\left[\mathrm{BrO}_{3}^{-}\right]>0.14 \mathrm{M}$ or whether it ends prematurely before reaching a homogeneously oxidized state (compare Figure 7a). The latter case would imply the existence of normal dispersion in the CHD-BZ system.

Figure $8 \mathrm{~b}$ shows that the stable period, $T_{0}$, decreases with increasing initial concentration of 1,4-CHD. At a critical concentration of $0.28 \mathrm{M}$, we observed the transition from wave stacking to merging. The monotonic decrease of $T_{0}$ with increasing values of [CHD] was confirmed for eight different concentrations of bromate (analyzed range, 0.06-0.16 M). Furthermore, all of these data sets showed the existence of a critical value of [CHD], above which wave merging is observed. The velocity data presented in Figure $7 \mathrm{~b}$ allows the straightforward conversion of stable periods $\left(T_{0}\right)$ to stable wavelengths $\left(\lambda_{0}=\mathrm{c} / T_{0}\right)$ and yields a monotonic decrease of $\lambda_{0}$ with

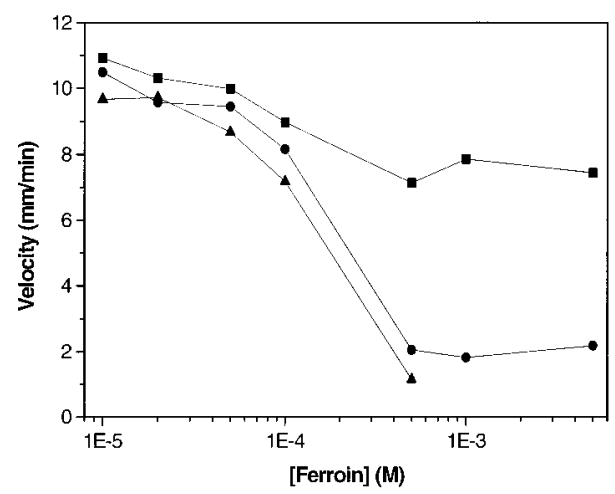

Figure 9. Wave velocities of solitary waves as a function of the initial ferroin concentration. The three graphs are obtained at different initial concentrations of 1,4-cyclohexanedione: 0.11 (squares), 0.19 (circles), and $0.29 \mathrm{M}$ (triangles) and at identical concentrations of $\mathrm{BrO}_{3}{ }^{-}(0.10$ M) and $\mathrm{H}_{2} \mathrm{SO}_{4}(2.0 \mathrm{M})$.

increasing values of [CHD] and a monotonic increase of $\lambda_{0}$ with increasing values of $\left[\mathrm{BrO}_{3}{ }^{-}\right]$. For the investigated range of concentrations (compare Figure 7), the stable wavelengths varied between 0.2 and $3.7 \mathrm{~mm}$.

The propagation velocity of waves in the $\mathrm{CHD}-\mathrm{BZ}$ reaction also depends on the initial concentration of the redox couple ferroin/ferriin. The half-logarithmic plot in Figure 9 shows that the wave speed decreases with increasing concentration of ferroin. The three curves in Figure 9 are typical examples that were selected from data obtained from a systematic survey of the wave speed at constant concentrations of sulfuric acid (2.0 $\mathrm{M})$ and bromate $(0.01 \mathrm{M})$. The typical shape of the corresponding curves is sigmoidal, with the most pronounced decrease occurring in the range of $0.1-1.0 \mathrm{mM}$. The overall magnitude of this ferroin-dependent change increases with increasing concentration of $\mathrm{CHD}$ and can reach factors of more than 5 . Wave propagation can become impossible in systems with high concentrations of CHD and ferroin. For example, we could neither observe nor initiate waves for reaction media with $\left[\mathrm{H}_{2} \mathrm{SO}_{4}\right]=2.0 \mathrm{M},\left[\mathrm{NaBrO}_{3}\right]=0.01 \mathrm{M},[\mathrm{CHD}]=0.29 \mathrm{M}$, and [ferroin] $>7 \times 10^{-4} \mathrm{M}$ (lowest curve in Figure 9). The pronounced dependence of the velocity on the initial concentration of ferroin shown in Figure 9 differs significantly from the behavior of classical BZ waves because in the latter case the velocity of ferroin and $\mathrm{Ru}(\mathrm{bpy})_{3}{ }^{2+}$ fronts is independent of the catalyst concentration. ${ }^{39,40}$ Moreover, in the cerium-catalyzed $\mathrm{BZ}$ reaction, the wave speed increases with increasing concentration of the catalyst. ${ }^{41}$

For the example of oscillations in well-stirred CHD-BZ systems, the complex role of ferroin has been discussed in earlier literature. Kurin-Csörgei et al. ${ }^{33}$ found that the reaction behaves like a typical BZ oscillator if the concentration of ferroin exceeds $5 \times 10^{-5} \mathrm{M}$. At concentrations of ferroin below $5 \times 10^{-5} \mathrm{M}$, however, it resembles an uncatalyzed bromate oscillator (UBO). The results presented in Figure 9 agree well with this finding because they reveal a marked change in the velocity of the first oxidation wave for concentrations of ferroin greater than $1 \times$ $10^{-4} \mathrm{M}$.

Dynamics at High Ferroin Concentration. Several earlier studies have investigated the dispersion behavior of the classical $\mathrm{BZ}$ reaction which employs malonic acid in the place of 1,4cyclohexanedione. ${ }^{16-18}$ These studies revealed satisfying agreement between experimental data and numerical results that were obtained from the Tyson-Fife model. ${ }^{16,42}$ This two-component model considers ferriin (the oxidized form of ferroin) to be the essential control variable which regulates the speed of the 


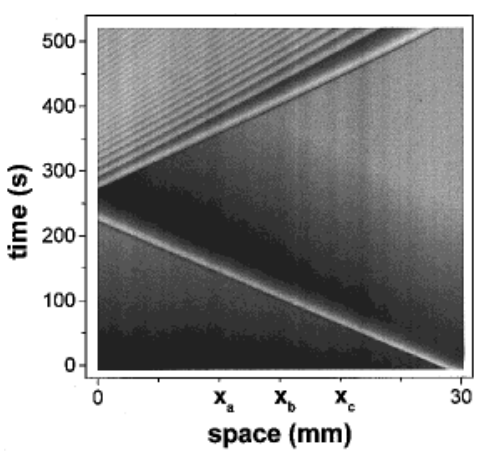

Figure 10. Time-space plot of the $\mathrm{CHD}-\mathrm{BZ}$ system at a high initial concentration of ferroin. Initial concentrations: $\left[\mathrm{H}_{2} \mathrm{SO}_{4}\right]=2.0 \mathrm{M}$, $\left[\mathrm{NaBrO}_{3}\right]=0.14 \mathrm{M},[1,4-\mathrm{CHD}]=0.19 \mathrm{M}$, and [ferroin $]=5 \mathrm{mM}$. The positions $x_{\mathrm{a}}, x_{\mathrm{b}}$, and $x_{\mathrm{c}}$ refer to the data shown in Figure 11.

propagating $\mathrm{HBrO}_{2}$ pulses. We therefore performed numerous experiments at high initial concentrations of ferroin to evaluate the relevance of ferriin as a direct control variable in the CHD$\mathrm{BZ}$ reaction.

Figure 10 shows a time-space plot of a pseudo-onedimensional CHD-BZ system at a high ferroin concentration of $5 \mathrm{mM}$. In this experiment, the first oxidation wave propagates with a constant velocity from the right to the left. This front is followed by several other waves that travel in the opposite direction. In the wake of the first pulse, the concentration of ferriin decreases rapidly to a very small value (dark areas in Figure 10). On a time scale of about $300 \mathrm{~s}$, however, the concentration of ferriin slowly increases again and creates, supposedly, a less favorable ferriin concentration in front of the following oxidation pulse. Nevertheless, the data given in Figure 10 clearly show that the propagation velocity of the second pulse is not affected by this steady increase. This observation is further confirmed by the dynamics of the first front which also encounters different concentrations of ferriin. In addition, the plot reveals a black boundary zone that precedes the rapid oxidation of ferroin in the $\mathrm{HBrO}_{2}$ pulse. This zone appears as a dark line in the time-space plot and appears in this experiment only for the first two waves which travel into (the bright) regions of high ferriin concentration.

To further quantify this unusual behavior, we analyzed the temporal evolution of the intensities for three different positions in the system. These points are labeled as $x_{\mathrm{a}}, x_{\mathrm{b}}$, and $x_{\mathrm{c}}$ in Figure 10 and correspond to the intensity data shown in panels $\mathrm{a}-\mathrm{c}$ Figure 11, respectively. The intensity values equal the monitored gray levels which are a linear measure for the local concentration of ferriin at any given time. The first peak in the three graphs is caused by the first oxidation wave of Figure 10. Its shape is characterized by a sequence of events that lead the system through a small initial drop, a rapid increase and a subsequent smooth decay in ferriin concentration. For the first (leftward propagating) wave, this complex kinetic behavior is most striking in Figure 11a, where the front encounters high concentrations of ferriin. However, the correlation between the amplitude of the initial dip and the prevailing concentration of ferriin is also apparent from the dynamics of the second (rightward propagating) pulse. Figure $11 \mathrm{c}$ reveals another interesting feature of the underlying kinetics. In this position, the slow relaxation process has sufficient time to fully unfold before the arrival of the next wave and the system undergoes a nearly linear increase in ferriin concentration that terminates at an about half-maximal plateau.

The data presented in Figures 10 and 11 differ significantly from the behavior of the classical BZ reaction which shows
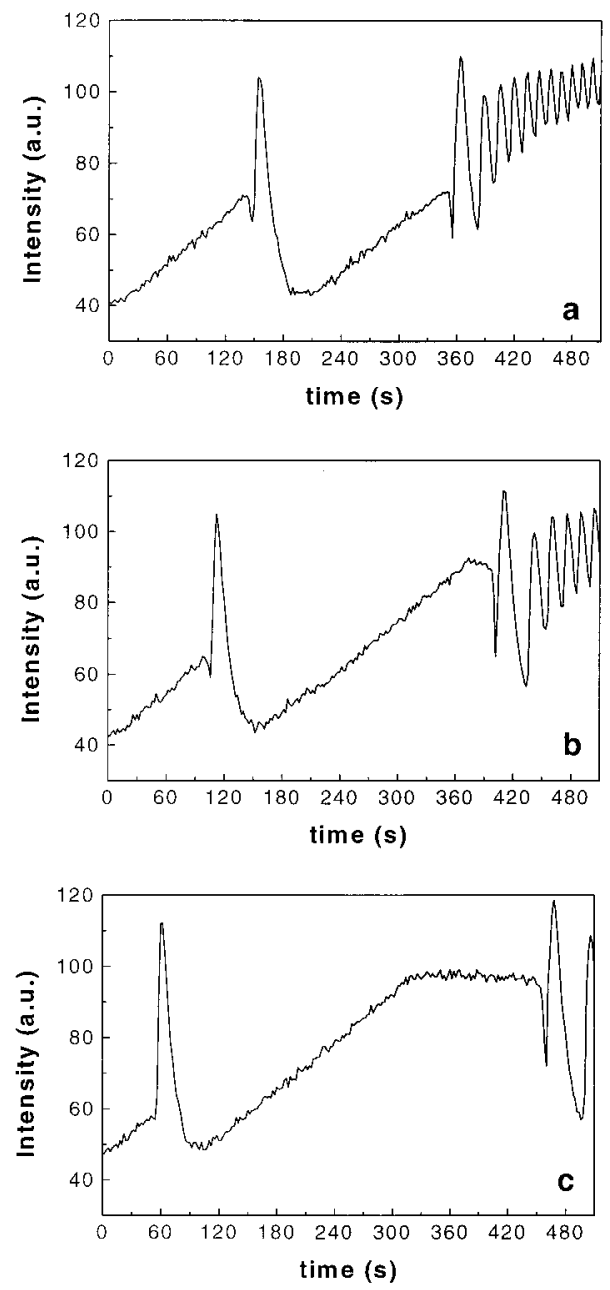

Figure 11. Temporal intensity profiles of the experiment shown in Figure 10. The three intensity profiles $\mathrm{a}, \mathrm{b}$, and $\mathrm{c}$ correspond to the positions $x_{\mathrm{a}}, x_{\mathrm{b}}$ and $x_{\mathrm{c}}$ of Figure 10, respectively.

neither the initial dip nor the slow reappearance of ferriin in the wake of the pulse. Furthermore, the results reveal that ferriin does not act as the sole control species in the $\mathrm{CHD}-\mathrm{BZ}$ reaction because the wave velocity can remain constant for different prevailing concentrations of the oxidized iron complex. Two features, however, are shared by the malonic acid and the 1,4cyclohexanedione system. In both systems, the ferriin profiles of oxidation pulses show a steep increase that is followed by a smooth decay in the wave back.

\section{Discussion}

Our study provides the first experimental proof for the existence of attractive pulse interaction in a homogeneously catalyzed reaction-diffusion system. The results rule out the involvement of reaction-induced convection, three-dimensional effects, and heterogeneities of the medium. The observed wave dynamics solely result from a complex relaxation process in the back of traveling oxidation pulses, which manifests itself in anomalous dispersion relations. Attractive pulse interaction had been predicted by numerous theoretical investigations ${ }^{12,21-26}$ but was not accessible to experiments due to the lack of appropriate chemical reaction systems.

In the $\mathrm{CHD}-\mathrm{BZ}$ reaction, one observes at least two qualitatively different modes of wave dynamics. The first one induces the formation of wave packets that are closely stacked at a constant period $T_{0}$. This period acts as an attractor which provides the dynamic stability of the stacked wave packet. 
Stacking behavior is characteristic for systems with nonmonotonic dispersion relations in which the slope of the function $c(T)$ is positive for small periods but negative for large periods. For these conditions, we also observed the bunching instability. Here, an unstable wave train undergoes a cascade of stacking events which create stable wave packets at marginally unstable distances. The second mode of wave dynamics leads to the merging of subsequent oxidation waves in front-to-back collisions. The underlying dispersion curve has a negative slope down to very small periods and shows no attractors. Most likely, the $\mathrm{CHD}-\mathrm{BZ}$ reaction shows also pure normal dispersion (i.e., $\mathrm{d} c / \mathrm{d} T \geq 0$ for allowed periods), which corresponds to repulsive wave interaction only.

The data presented in this paper reveal the following bifurcation sequence that occurs in response to a decrease (increase) of the initial concentration of bromate (CHD): (1) homogeneously oxidized, (2) normal dispersion, (3) wave stacking, (4) wave merging, and (5) homogeneously reduced. In the transition from 2 to 3 , the normal dispersion curve develops an overshoot at a very large or possibly infinite period. This process creates a unique attractor $\left(T_{0}, c_{0}\right)$, and wave stacking can take place. With decreasing concentration of bromate, the overshoot and the value of $T_{0}$ shift toward lower periods until a critical minimum is reached at which the stacking/ merging bifurcation occurs. This sequence is furthermore accompanied by a steady decrease in the speed of solitary waves $\left(c_{0}\right)$. The same changes of the dispersion curve occur with increasing concentration of 1,4-cyclohexanedione.

The qualitative behavior of waves in the CHD-BZ reaction is in excellent agreement with earlier predictions that were derived from pairs of coupled reaction-diffusion equations such as the FitzHugh-Nagumo or the Hodgkin-Huxley model. ${ }^{12,21-23}$ However, anomalous dispersion in this experimental system stems from the interplay of $\mathrm{HBrO}_{2}$ with at least two independent species that control the relaxation of the medium in the wake of traveling pulses. The dependence of the wave dynamics on the initial concentration of ferroin yields some crucial insights into the structure of the underlying reaction mechanism. Our results (Figure 9) clearly indicate that the CHD-BZ reaction is ferroin-controlled only for initial concentrations of ferroin that exceed $1 \times 10^{-3} \mathrm{M}$. For concentrations around $1 \times 10^{-5}$ $\mathrm{M}$ and smaller, the systems shows the characteristics of an uncatalyzed bromate oscillator $\left(\mathrm{UBO}^{5,43}\right)$. Most of the wave experiments in this study, however, are carried out at a ferroin concentration of $5 \times 10^{-4} \mathrm{M}$ and fall into the transitional range that involves control by ferroin as well as control by a derivative of 1,4-CHD. Therefore, anomalous dispersion behavior could arise from the inhibiting action of ferriin in the immediate vicinity of the $\mathrm{HBrO}_{2}$ pulse and the activating effect of a CHDderived intermediate at farther distances. Here, the local concentration gradients of the involved (activating and inhibiting) species determine a gradient in wave velocity that induces acceleration at intermediate distances and deceleration close to the leading wave. At large distances, this velocity gradient is nearly zero, and hence, no significant wave acceleration is observed

Several earlier studies were dedicated to the identification of intermediate species and products in the reaction of 1,4-CHD with acidic bromate. ${ }^{44,45}$ It was found that 1,4-dihydroxybenzene $\left(\mathrm{H}_{2} \mathrm{Q}\right)$ is formed and further oxidized and brominated to 1,4benzoquinone and bromoorganics. Szalai et al. pointed out the importance of the rather stable oxidation product $\mathrm{H}_{2} \mathrm{Q}$, which is produced continuously but oxidized in an autocatalytic fashion. ${ }^{45,46}$ In contrast to the intensively studied CHD UBO, little is known about the $\mathrm{CHD}-\mathrm{BZ}$ mechanism and its dynamic behavior. The latter one is more complex because it involves additional reaction such as the ones between $\mathrm{CHD}, \mathrm{H}_{2} \mathrm{Q}, \mathrm{HBrO}_{2}$, and ferriin. Additional studies will be necessary to clarify the kinetics of the $\mathrm{CHD}-\mathrm{BZ}$ reaction and to formulate an appropriate reaction model that is capable of reproducing the observed wave phenomena.

The impact of this study extends beyond the demonstration and characterization of anomalous dispersion in a liquid phase reaction. Our results lay the foundation for a systematic investigation of anomalous dispersion in excitable systems that will improve our knowledge of this widely unexplored aspect of chemical pattern formation. In particular, it will be interesting to analyze the consequences of attractive pulse interaction (i.e., stacking, merging, and bunching) in spatially two-dimensional media and to test predictions concerning the formation of waveemitting fronts (WEFs) that give rise to spatiotemporal chaos. ${ }^{29,47}$ Moreover, the experimental investigation of the CHD-BZ reaction should foster our understanding of stable pulse propagation in neuronal systems. Here, recent results challenge the classical view of neural coding by the rate of discharge events and suggest that information is transmitted in precisely timed trains of action potentials. ${ }^{48,49}$ Since the propagation of excitation pulses in neuronal systems can obey anomalous and even oscillatory dispersion relations, ${ }^{48}$ the resulting stable wave packets could be essential for reliable transport and encoding of information.

Acknowledgment. This work was financially supported by The Florida State University. We thank K. Agladze and M. Hildebrand for fruitful discussions.

\section{References and Notes}

(1) Nicolis, G.; Prigogine, I. Self-Organization in Nonequilibrium Systems; Wiley-Interscience: New York, 1977.

(2) Kapral, R.; Showalter, K., Eds. Chemical Waves and Patterns; Kluwer: Dordrecht, The Netherlands, 1995. 1173.

(3) Merzhanov, A. G.; Rumanov, E. N. Rev. Mod. Phys. 1999, 71,

(4) Ross, J.; Müller, S. C.; Vidal, C. Science 1988, 240, 460

(5) Epstein, I. R.; Pojman, J. A. An Introduction to Nonlinear Chemical Dynamics; Oxford University Press: New York, 1998.

(6) Scott, S. K.; Johnson, B. R.; Taylor, A. F.; Tinsley, M. R. Chem Eng. Sci. 2000, 55, 209.

(7) Rotermund, H. H. J. Electron Spectrosc. 1999, 99, 41.

(8) Agladze, K.; Steinbock, O. J. Phys. Chem. A 2000, 104, 9816.

(9) Müller, S. C.; Mair, T.; Steinbock, O. Biophys. Chem. 1998, 72, 37.

(10) Jung, P.; Cornell-Bell, A.; Madden, K. S.; Moss, F. J. Neurophysiol. 1998, 79, 1098 .

(11) Hess, B. Naturwissenschaften 2000, 87, 199.

(12) Rinzel, J.; Maginu, K. In Nonequilibrium Dynamics in Chemical Systems; Vidal, C., Pacault, A., Eds.; Springer: Berlin, 1984; pp 107113.

(13) Winfree, A. T. Chaos 1991, 1, 303.

(14) Dockery, J. D.; Keener, J. P.; Tyson, J. J. Physica D 1988, 30 , 177.

(15) Dockery, J. D.; Keener, J. P. SIAM J. Appl. Math. 1989, 49, 539.

(16) Pagola, A.; Ross, J.; Vidal, C. J. Phys. Chem. 1988, 92, 163

(17) Steinbock, O.; Müller, S. C. Physica A 1992, 188, 61.

(18) Flesselles, J. M.; Belmonte, A.; Gaspar, V. J. Chem. Soc., Faraday Trans. 1998, 94, 851.

(19) Nassar, S. F.; Ismail, H. A.; Sayed, E. M.; El-Falaky, A. Appl. Phys. 1975, 7, 307.

(20) Siegert, F.; Weijer, C. J. Cell Sci. 1989, 93, 325.

(21) Elphick, C.; Meron, E.; Spiegel, E. A. Phys. Rev. Lett. 1988, 61, 496.

(22) Elphick, C.; Meron, E.; Spiegel, E. A. SIAM J. Appl. Math. 1990, 50,490 .

(23) Elphick, C.; Meron, E.; Rinzel, J.; Spiegel, E. A. J. Theor. Biol. 1990, 146,249

(24) Winfree, A. T. Phys. Lett. 1990, 149, 203.

(25) Winfree, A. T. Physica D 1991, 49, 125. 
(26) Or-Guil, M.; Kevrekidis, I. G.; Bär, M. Physica D 2000, 135, 154.

(27) Siegert, F.; Weijer, C. J. Physica D 1991, 49, 224.

(28) Christoph, J.; Eiswirth, M.; Hartmann, N.; Imbihl, R.; Kevrekidis, I.; Bär, M. Phys. Rev. Lett. 1999, 82, 1586.

(29) Manz, N.; Müller, S. C.; Steinbock, O. J. Phys. Chem. A 2000, 104,5895

(30) Kurin-Csörgei, K.; Zhabotinsky, A. M.; Orbán, M.; Epstein, I. R. J. Phys. Chem. A 1997, 101, 6827.

(31) Davydov, V. A.; Manz, N.; Steinbock, O.; Zykov, V. S.; Müller, S. C. Phys. Rev. Lett. 2000, 85, 868.

(32) Müller, S. C.; Plesser, T. Hess, B. Physica 1987, D24, 71.

(33) Kurin-Csörgei, K.; Zhabotinsky, A. M.; Orbán, M.; Epstein, I. R. J. Phys. Chem. 1996, 100, 5393.

(34) Steinbock, O.; Hamik, C. T.; Steinbock, B. J. Phys. Chem. A 2000, 104,6411 .

(35) Komlósi, A.; Nagy, I. P.; Bazsa, G.; Pojman, J. A. J. Phys. Chem. A 1998, 102, 9136 .

(36) Diewald, M.; Brand, H. R. Phys. Rev. E 1995, 51, R5200.

(37) Chandrasekhar, S. Hydrodynamic and Hydromagnetic Stability; Oxford University Press: London, 1961.
(38) Belmonte, A. L.; Ouyang, Q.; Flesselles, J.-M. J. Phys. II France 1997, 7, 1425.

(39) Field, R. J.; Noyes, R. M. J. Am. Chem. Soc. 1974, 96, 2001.

(40) Kuhnert, L.; Krug, H.-J. J. Phys. Chem. 1987, 91, 730.

(41) Nagy-Ungvarai, Z.; Tyson, J. J.; Hess, B. J. Phys. Chem. 1989, 93, 707 .

(42) Keener, J. P.; Tyson, J. J. Physica D 1986, 21, 307.

(43) Orbán, M.; Kurin-Csörgei, K.; Zhabotinsky, A. M.; Epstein, I. R. J. Am. Chem. Soc. 1998, 120, 1146.

(44) Kurin-Csörgei, K.; Szalai, I.; Molnarperl, I.; Körös, E. React. Kinet. Catal. Lett. 1994, 53, 115.

(45) Szalai, I.; Körös, E. J. Phys. Chem. A 1998, 102, 6892.

(46) Szalai, I.; Körös, E.; Györgyi, L. J. Phys. Chem. A 1999, 103, 243.

(47) Zimmermann, M. G.; Firle, S. O.; Natiello, M. A.; Hildebrand, M.; Eiswirth, M.; Bär, M.; Bangia, A. K.; Kevrekidis, I. G. Physica D 1997, 110,92 .

(48) Brenner, N.; Agam, O.; Bialek, W.; de Ruyter van Steveninck, R. R. Phys. Rev. Lett. 1998, 81, 4000 .

(49) Diesmann, M.; Gewaltig, M.-O.; Aertsen, A. Nature 1999, 402, 529 . 